\title{
Human metabolic response to systemic inflammation: assessment of the concordance between experimental endotoxemia and clinical cases of sepsis/SIRS
}

Kubra Kamisoglu', Beatrice Haimovich², Steve E Calvano², Susette M Coyle², Siobhan A Corbett ${ }^{2}$, Raymond J Langley ${ }^{3}$, Stephen F Kingsmore ${ }^{4,5}$ and loannis P Androulakis ${ }^{1,2,6^{*}}$

\begin{abstract}
Introduction: Two recent, independent, studies conducted novel metabolomics analyses relevant to human sepsis progression; one was a human model of endotoxin (lipopolysaccharide (LPS)) challenge (experimental endotoxemia) and the other was community acquired pneumonia and sepsis outcome diagnostic study (CAPSOD). The purpose of the present study was to assess the concordance of metabolic responses to LPS and community-acquired sepsis.

Methods: We tested the hypothesis that the patterns of metabolic response elicited by endotoxin would agree with those in clinical sepsis. Alterations in the plasma metabolome of the subjects challenged with LPS were compared with those of sepsis patients who had been stratified into two groups: sepsis patients with confirmed infection and non-infected patients who exhibited systemic inflammatory response syndrome (SIRS) criteria. Common metabolites between endotoxemia and both these groups were individually identified, together with their direction of change and functional classifications.
\end{abstract}

Results: Response to endotoxemia at the metabolome level elicited characteristics that agree well with those observed in sepsis patients despite the high degree of variability in the response of these patients. Moreover, some distinct features of SIRS have been identified. Upon stratification of sepsis patients based on 28-day survival, the direction of change in 21 of 23 metabolites was the same in endotoxemia and sepsis survival groups.

Conclusions: The observed concordance in plasma metabolomes of LPS-treated subjects and sepsis survivors strengthens the relevance of endotoxemia to clinical research as a physiological model of community-acquired sepsis, and gives valuable insights into the metabolic changes that constitute a homeostatic response. Furthermore, recapitulation of metabolic differences between sepsis non-survivors and survivors in LPS-treated subjects can enable further research on the development and assessment of rational clinical therapies to prevent sepsis mortality. Compared with earlier studies which focused exclusively on comparing transcriptional dynamics, the distinct metabolomic responses to systemic inflammation with or without confirmed infection, suggest that the metabolome is much better at differentiating these pathophysiologies. Finally, the metabolic changes in the recovering patients shift towards the LPS-induced response pattern strengthening the notion that the metabolic, as well as transcriptional responses, characteristic to the endotoxemia model represent necessary and "healthy" responses to infectious stimuli.

\footnotetext{
*Correspondence: yannis@rci.rutgers.edu

'Department of Chemical and Biochemical Engineering, Rutgers University,

Piscataway, NJ 08854, USA

2Department of Surgery, Rutgers - Robert Wood Johnson Medical School,

New Brunswick, NJ 08901, USA

Full list of author information is available at the end of the article
}

\section{Ciomed Central}

(c) 2015 Kamisoglu et al.; licensee BioMed Central. This is an Open Access article distributed under the terms of the Creative Commons Attribution License (http://creativecommons.org/licenses/by/4.0), which permits unrestricted use, distribution, and reproduction in any medium, provided the original work is properly credited. The Creative Commons Public Domain Dedication waiver (http://creativecommons.org/publicdomain/zero/1.0/) applies to the data made available in this article, unless otherwise stated. 


\section{Introduction}

Sepsis is defined as the combination of an infection with multiple features of 'systemic inflammatory response syndrome' (SIRS) [1] and is one of the oldest and most enigmatic conditions in medicine. There are more than a million cases of sepsis per year in the United States [2] and it is estimated that there are 19 million cases per year worldwide [3,4]. According to the Centers for Disease Control, the cost of hospitalization is in the order of $\$ 15$ billion, with an anticipated further increase in the future [5]. Despite several decades of intensive research and efforts to bring new therapies to the bedside, the number of cases and sepsis-associated deaths are still soaring $[3,6]$. Current treatment guidelines include cardiorespiratory resuscitation and non-specific protocols aimed at mitigating immediate threats of uncontrolled infection [3]. A significant barrier to progress is the perceived inadequacy of experimental models that can reproduce the pathophysiology of the disease in humans.

The high degree of variability among patients and multiple aspects of the disease, including patient gender, age and comorbidities complicate the design of relevant experimental models and clinical studies. Moreover, the initiating cause of infection and the physiologic responses that follow are also highly variable [7]. All these factors explain, at least in part, the difficulty in translating experimental results to the clinic and, consequently, the lack of success in the development of effective therapies [8].

Endotoxemia, an experimental model in which healthy volunteers are intravenously administered a form of endotoxin (lipopolysaccharide, LPS, a major component of Gram-negative bacteria outer membrane and a Toll-like receptor 4 (TLR4) agonist) [9], has served as a valuable experimental venue for more than six decades [10-12]. It is a model of systemic inflammation, rather than a true mimic of sepsis. Nonetheless, early transient physiochemical changes and biochemical pathway activation in this model are strikingly similar to those observed during the early hyperdynamic phase of resuscitated injury and infection [13]. The LPS challenge triggers chills, myalgias, nausea, and an increase in core body temperature and heart rate, most of which begin to abate within six to eight hours $[11,13,14]$. Genome-wide analyses of circulating leukocytes revealed transcriptional signatures indicative of changes in protein translation and glycolysis [15], which shared similar characteristics with those observed in trauma patients [16]. These studies helped elucidate the intricate regulatory schemes governing the response to endotoxemia $[16,17]$ and provided the foundations for in silico models of systemic inflammation [18-24]. More recently, we documented the effects of LPS-induced inflammation on the whole body metabolism in humans [25]. In contrast with other methods applied to the endotoxemia model, metabolomics reflects the combined output of all tissues in the body [26]. In that study [25], plasma samples, collected from healthy subjects during 24-hours post challenge with LPS, were subjected to non-targeted biochemical profiling, revealing the temporal changes in the plasma metabolome. Unsupervised multivariate analyses identified prominent changes in lipid and protein metabolism, which peaked at six hours post LPS infusion. Subsequently, to understand better how the inflammatory responses at the level of cells and whole body correlate in humans, we integrated the analysis of the plasma metabolome with that of the leukocyte transcriptome [27].

In [28], an integrated analysis of clinical features, plasma metabolome and proteome described the pattern of metabolic perturbations in critically ill patients presenting with symptoms of SIRS or sepsis. This study, the first of its kind, examined clinical features as well as the plasma metabolome, and proteome, of patients upon arrival at the emergency department (ED) and 24 hours later. An important and novel outcome of the study was the realization that metabolic differences could ultimately be used as markers predicting survival.

Since the endotoxemia model utilizes LPS, rather than intact bacteria, there is an ongoing concern that data derived from this model are of limited relevance to our understanding of sepsis-induced inflammatory mechanisms, although recent analyses of the leukocyte transcriptome seemed to argue otherwise [15]. The availability of new metabolomic data $[25,28]$ offered the opportunity to compare responses detected in LPS-challenged subjects to those of critically ill patients at the level of the entire organism. In this retrospective study we aimed to objectively determine the relevance of the information content gained by parallel analyses of LPS-challenged subjects [25] and patients with or without communityacquired sepsis [28]. Our study identified a core response that was in agreement with what was observed in sepsis patients. Response to systemic inflammation without apparent infectious stimuli such as what is observed in SIRS was shown to have distinct features that may make it uniquely recognizable at the metabolomics level. Metabolic changes in the patients who are recovering shifted towards an endotoxemia response pattern, strengthening the idea that the endotoxemia model represents necessary and 'healthy' responses to an infectious stimulus.

\section{Material and methods}

\section{Metabolic data}

This is a retrospective analysis utilizing metabolomes obtained from subjects who participated in an experimental endotoxemia study and from patients with or without community-acquired sepsis. In brief, as previously described [25], healthy volunteers participated in an 
endotoxemia study, after providing written, informed consent under guidelines approved by the Institutional Review Board of Rutgers - Robert Wood Johnson Medical School. Inclusion criteria were age between 18 to 40 years, and normal general health as demonstrated by medical history and physical examination as well as laboratory testing. National Institutes of Health (NIH) Clinical Center Reference Endotoxin at a bolus dose of $2 \mathrm{ng} / \mathrm{kg}$ body weight was administered to 15 subjects. Blood samples were collected at $\mathrm{t}=0$, prior to treatment, and at 1, 2, 6, and 24 hours post-treatment (Figure 1, top). Mass spectrometry (MS) based biochemical analysis of the plasma samples was performed by Metabolon (Durham, NC, USA) according to previously published methods [29]. All details of the study design and biochemical analysis are available in [25].

Metabolomic data for the clinical cases of systemic inflammation were obtained from the Community Acquired Pneumonia and Sepsis Outcome and Diagnostics (CAPSOD) study [28]. Approval for this study was obtained by institutional ethics committees and details were filed at ClinicalTrials.gov (NCT00258869). Protocols and identified clinical features in the different classes of patients were previously published. The study [28] included 1,152 individuals with suspected, community-acquired sepsis (acute infection and $\geq 2$ SIRS criteria) in the emergency departments at three urban, tertiary-care hospitals in the United States between 2005 and 2009. Each patient or their legal designates provided informed consent. Medical history, physical examination, and acute illness scores (Acute Physiology and Chronic Health Evaluation II
(APACHE II) and Sequential Organ Failure Assessment (SOFA)) were recorded at enrollment $\left(\mathrm{t}_{0, \text { clinical }}\right)$ and 24 hours later $\left(\mathrm{t}_{24, \mathrm{clinical}}\right)$. Infection status and outcome through day 28 were independently determined by board-certified clinicians. Clinical care given for the patients was not standardized and was determined by individual providers. After independent audit of infection status and outcomes, 150 patients were chosen for derivation studies. Non-targeted mass spectrometry based analyses of the patients' blood samples were done by Metabolon similarly to the endotoxemia study.

The 150 patients chosen for derivation studies within the CAPSOD cohort were classified to represent cases of uncomplicated sepsis $(n=27)$, severe sepsis $(n=25)$, septic shock $(\mathrm{n}=38)$, non-infected SIRS ('ill' controls, indicated as SIRS, presumed septic at enrollment but later determined to have noninfectious reasons for SIRS; $\mathrm{n}=29$ ) or sepsis non-survival (SNS, $\mathrm{n}=31$ ) (Figure 1, bottom). No significant differences among subgroups of sepsis survivors (uncomplicated sepsis, severe sepsis, septic shock) were reported for plasma metabolites [28]. Therefore, the data from these patients were collapsed into a single group referred to as sepsis survivors $(\mathrm{SS}, \mathrm{n}=90)$ for the purposes of this study. Furthermore, in the first part of the analysis, metabolic data from SS and sepsis non-survivors (SNS) were pooled to assess the similarities and differences between sepsis and non-infected SIRS, and referred to as the Sepsis group $(n=121)$. In the subsequent analysis, data from the SS and SNS groups were used individually to investigate the association of metabolic changes in endotoxemia with

\section{Experimental Endotoxemia}

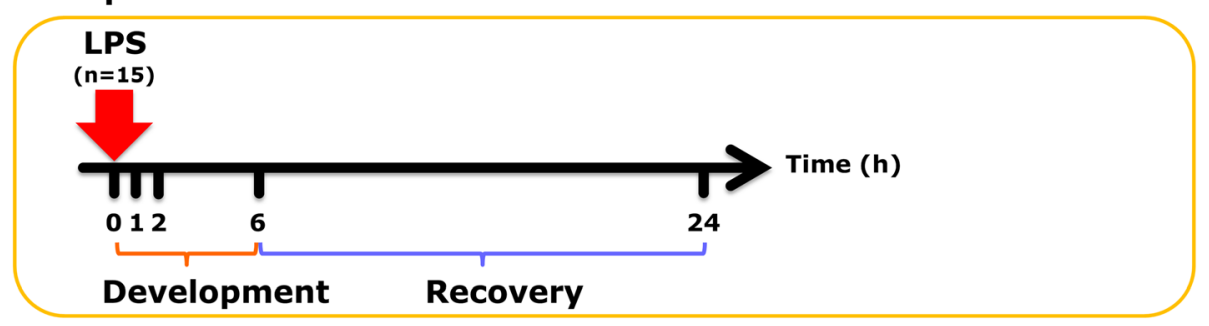

Clinical Cases of Systemic Inflammation

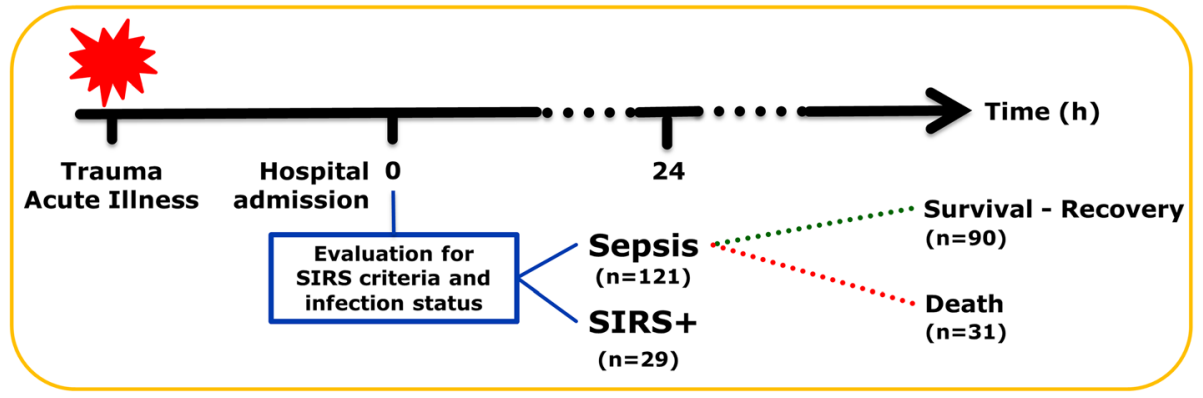

Figure 1 Schematic description of the experimental and clinical sources of data used. 
those observed in either surviving or non-surviving sepsis patients.

\section{Data analysis}

MS analysis of plasma samples from the human endotoxemia study provided temporal information on 366 metabolites at five time points. Previous results of the principal component analysis on this dataset showed that the six hour time point $\left(\mathrm{t}_{6}\right)$ was the most critical since the maximum difference between control and treatment groups was observed at this time point [25]. This agreed well with prior transcriptional studies indicating that the maximal change in leukocyte gene expression was observed six hours after the LPS administration [16,30]. Therefore, this data point was considered to represent the peak of metabolic response to endotoxemia and used as reference for the assessment of concordance between experimental and clinical data in this study. MS analysis of the samples from the CAPSOD study, on the other hand, had identified 370 metabolites at $t_{0, c l i n i c a l}$ (time of hospital admission) and 401 metabolites at $t_{24, c l i n i c a l}$ (24 hours after admission). In this study, both clinical and experimental datasets were individually normalized by setting the median equal to 1 . Missing values were imputed with the observed minimums after normalization. The metabolite lists were consolidated. Only the metabolites commonly identified in the endotoxemia [25] and clinical [28] studies were analyzed further. The final dataset included 177 common metabolites from both studies [25,28]. Outliers were removed using the median absolute deviation, $\mathrm{MAD}=1.4826 \times \mid\left(\mathrm{x}_{\mathrm{i}}-\mathrm{Median}_{\mathrm{j}}\left(\mathrm{x}_{\mathrm{j}}\right) \mid\right.$, of each metabolite, determined in each individual group $[31,32]$. Subsequently, the score for each data point was calculated $z_{i}=\frac{\left|x_{i}-\operatorname{Median}_{j}\left(x_{j}\right)\right|}{\operatorname{MAD}}$ and data points with a score above 3 were removed from the dataset. The number of removed outliers for each group is reported in Additional file 1: Table S1.

The baseline of the human endotoxemia studies, that is, samples collected before LPS administration $\left(t_{0, L P S}\right)$, defined the 'baseline' in this study. We identified the six hour time point as the peak of the metabolic response in the endotoxemia model in our previous metabolomics study [25], as well as transcriptomic analysis [17,18], and hypothesized that this time point represents the point of transition from the development and recovery phases of the response. For the clinical data collection, the starting point was the time of hospital admission $\left(t_{0, \text { clinical }}\right)$, whereas the second clinical time point was 24 hours later $\left(t_{24, \text { clinical }}\right)$. Since the data obtained for the clinical patients lack internal controls, for obvious reasons, the responses of each group of patients, as well as the endotoxemia subjects, were compared independently to the healthy baseline $\left(t_{0, \mathrm{LPS}}\right)$. For comparing the means of metabolites in each condition relative to the healthy baseline, Welch's t-test was used without assuming equal variances $(\alpha=0.05)$. The number of subjects in the experimental endotoxemia group $(n=15)$ was much smaller than the number of patients in the clinical groups to assume normal distribution required for the $\mathrm{t}$-test. However, at both $t_{0, L P S}$ and $t_{6, L P S}$, the data passed the Kolmogorov-Smirnov test for each metabolite allowing the application of the t-test. Q-values were calculated according to the Benjamini and Hochberg procedure [33] and metabolites having a $P$ - and a q-value less than 0.05 are called significant. We also evaluated how dispersed the data for each metabolite is in clinical cases with respect to those at the baseline. Variances of the significant metabolites in each condition were also plotted relative to the variances at the baseline and are shown in Additional file 2: Figure S1. The direction and magnitude of changes in plasma metabolite concentrations were determined based on $\log 2$ fold changes from $t_{0, L P S}$ for both clinical groups and plotted against those observed for endotoxemia as shown in Figure 2. The full list of the metabolites, their significance in each condition, the direction and magnitude of the changes relative to the baseline is provided in Additional file 3: Table S2.

We lastly focused on changes in metabolites within subpopulations of sepsis patients who ultimately survived, and those who did not. For this purpose, dynamics within the sepsis group were examined using the data from the SS and SNS groups. Metabolites that statistically differed between these groups at either time point were determined by $\mathrm{t}$-test as described earlier. The magnitude of changes in plasma metabolites relative to $t_{0, \mathrm{LPS}}$ were calculated for each group. These changes were compared between the SS and SNS groups at both time points and with the endotoxemia group at $t_{6, \text { LPS }}$

\section{Results and discussion}

Endotoxemia induced by elective administration of LPS to healthy subjects has served as an invaluable tool for obtaining mechanistic insight into homeostatic inflammatory responses. Previous studies compared transcript- and protein-expression patterns in immune cells obtained from LPS treated subjects and trauma patients, revealing significant overlap [15,34]. More recently, metabolomics analyses in both LPS-administered subjects [25] and patients with symptoms of systemic inflammation at time of presentation to emergency departments were published $[28,35]$. Building on these prior studies $[26,29]$, here we aimed to objectively compare metabolic indices obtained from experimental studies and clinical sources.

The inherent dynamics of a clinical and an experimental study are obviously disparate although they focus on related physiologic phenomena. The first time point in a clinical study is generally at a point that had already deviated from what can be called a 'healthy state,' whereas experimental 
A Endotoxemia vs. Sepsis

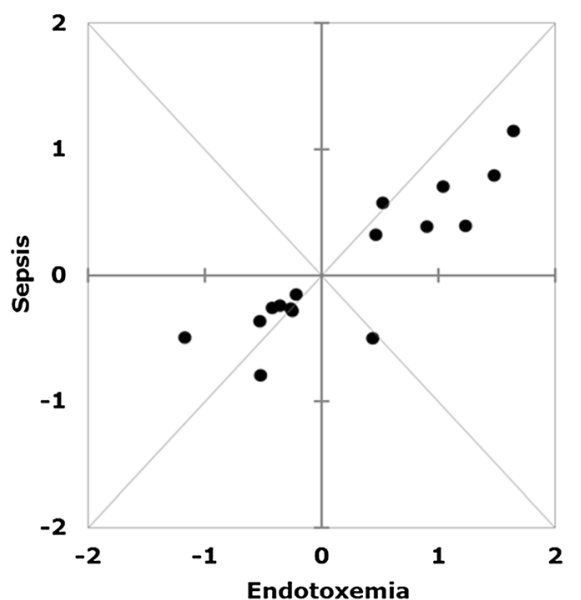

\section{B Endotoxemia vs. SIRS}

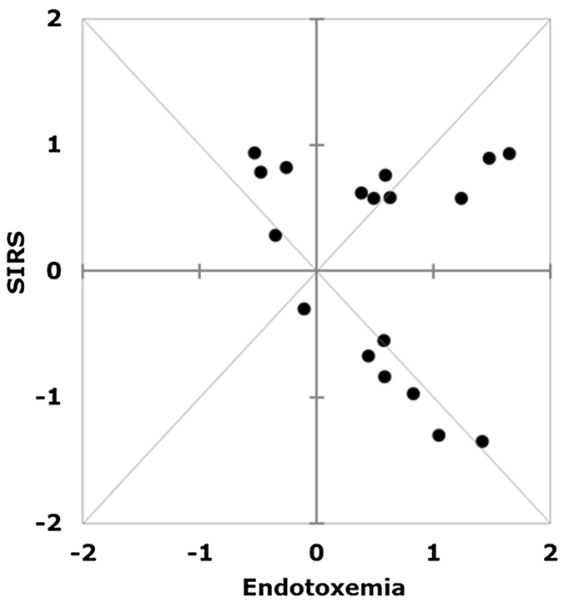

Figure 2 Scatter plots show the direction and extent of changes in the metabolites that significantly deviated from baseline in sepsis (A) and SIRS (B) groups in relation to corresponding trends in endotoxemia. For the clinical data, plots reflect the maximum observed change from the baseline if that particular metabolite was found to be significant in both time points, $t_{0, c l i n i c a l}$ and $t_{24, c l i n i c a l}$. SIRS, severe inflammatory response syndrome.

studies usually measure divergence from the 'healthy state' under controlled conditions. In this study, we aimed to evaluate the significance of observed metabolic perturbations in endotoxemia and how they relate to corresponding changes observed in patients with symptoms of community-acquired sepsis at the time of presentation to the emergency departments. In line with our objective, we chose to evaluate each condition and each time point based on its deviation from one common baseline that reflects a healthy state $\left(t_{0, \text { LPS }}\right)$. Table 1 shows the number of metabolites that had a significantly different concentration compared to $t_{0, L P S}$ at each time point available for each condition. The first column in Table 1, shows the total information content of the final consolidated dataset with total number of metabolites associated with each metabolic super-pathway. The complete list of metabolites, their pathway classification, and extent of changes from baseline are provided in Additional file 3: Table S2. At the peak of the response to LPS, that is, at $t_{6, \mathrm{LPS}}$, there were 83 metabolites (47\% of the total 177 common metabolites) which significantly deviated from baseline. In contrast, the number of metabolites that significantly differed from baseline for the clinical groups was considerably smaller (varying between 19 to 26 metabolites, or $11 \%$ to $15 \%$ of the total 177 common metabolites). We hypothesize that the much larger number of metabolites that changed significantly in response to endotoxin as compared to the clinical cases

Table 1 Number of significantly changed metabolites and metabolic super-pathways that they belong to, determined for LPS-challenged subjects and patient groups

\begin{tabular}{|c|c|c|c|c|c|c|}
\hline \multirow[b]{2}{*}{ Super pathway } & \multirow[b]{2}{*}{ Total } & \multirow{2}{*}{$\begin{array}{l}\text { LPS (number = 15) } \\
\text { t6 }\end{array}$} & \multicolumn{2}{|c|}{ Sepsis (number $=121$ ) } & \multicolumn{2}{|c|}{ SIRS (number = 29) } \\
\hline & & & to & $\mathrm{t} 24$ & to & $\mathrm{t} 24$ \\
\hline Amino acid & 55 & 28 & 3 & 5 & 3 & 4 \\
\hline Carbohydrate & 16 & 4 & 1 & 2 & 2 & 2 \\
\hline Cofactors and vitamins & 7 & 3 & 2 & 1 & 2 & 1 \\
\hline Lipid & 75 & 36 & 10 & 16 & 12 & 16 \\
\hline Energy & 4 & 4 & 0 & 1 & 1 & 1 \\
\hline Nucleotide & 8 & 3 & 0 & 0 & 1 & 0 \\
\hline Peptide & 3 & 3 & 0 & 0 & 0 & 0 \\
\hline Xenobiotics & 9 & 2 & 3 & 1 & 0 & 1 \\
\hline Total & 177 & 83 & 19 & 26 & 21 & 25 \\
\hline
\end{tabular}

Significance was determined by comparing responses of each group of patients, as well as endotoxemia subjects, to the healthy baseline ( $t_{0, L P S}$ ) individually. Welch's t-test was used and with correction for multiple comparisons by the Benjamini and Hochberg procedure. $(a=0.05)$. Metabolites having a $P$ - and a q-value less than 0.05 are called significant. (The complete list is available in Additional file 3: Table S2). LPS, lipopolysaccharide; SIRS, severe inflammatory response syndrome. 
reflects, at least in part, the fundamental difference between physiologic variability of responses elicited in subjects who participated in the controlled endotoxemia study and patients. The endotoxemia study cohort included relatively young and healthy subjects whereas the patient cohort that participated in the CAPSOD study was variable, in terms of age and comorbidities among others. In addition, the trigger itself, that is, LPS, activates a single TLR4-dependent signaling pathway, whereas infectious agents and trauma activate multiple ones, leading to greater variability in responses. In order to evaluate the level of dispersion in the clinical data with respect to the experimental, the variance of each significant metabolite at each clinical condition was calculated and plotted against the corresponding variance at the baseline. These plots are show in Additional file 2: Figure S1. As highlighted in these plots, variances of the metabolites measured in the patients were statistically higher than those measured in the endotoxemia study participants at the baseline, $t_{0, L P S}$, reflecting the fundamental differences in variability between the two groups.

Next, we sought to determine the similarities and differences among the subsets of significant metabolites that changed significantly in the sepsis and SIRS groups which were also significant in endotoxemia. Our intention was to be maximally inclusive of the clinically observed changes. Therefore, we focused on metabolites that were significantly different from the baseline at either one of the two clinical time points as well as in endotoxemia. Table 2A lists the metabolites common to endotoxemia and sepsis and Table 2B lists those common to endotoxemia and SIRS. Metabolites that are common to both lists A and $\mathrm{B}$ are typed in bold. Triangles depict the direction (apex up: increase, apex down: decrease) and magnitude (one triangle: less than two fold change, two triangles: more than two fold change) of the difference relative to the baseline $\left(t_{0, L P S}\right)$. Although the total number of metabolites in common with endotoxemia is close for the two clinical cases (16 in Table 2A and 18 in Table 2B), the agreement between the directions of change is strikingly different. Bilirubin, docosapentaenoate (DPA) and palmitolate were the only three metabolites common to the LPS, sepsis and SIRS groups, which changed in the same direction. Of the 16 metabolites common to LPS and sepsis (Table 2A), 15 changed in the same direction. Only one, xylose, changed in an opposite direction. In marked contrast, of the total 18 metabolites common to the endotoxemia and SIRS groups, 10 changed in the opposite direction (Table 2B). Scatter plots shown in Figure 2A and B highlight this distinction in response. The axes of the scatter plots indicate the $\log 2$ fold changes in metabolite concentrations. The $\mathrm{x}$-axes show the change at $\mathrm{t}_{6, \mathrm{LPS}}$ from baseline, $\mathrm{t}_{0, \mathrm{LPS}}$. The y-axes show the maximum change in the clinical data

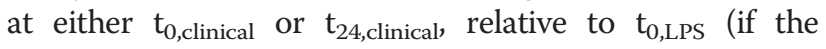

changes at both time points were significant, the higher of the two values is shown). Positive direction shows an increase in concentration, while negative shows a decrease. Accordingly, the concentrations of metabolites in the first and third quadrants change in parallel with the observations in endotoxemia; while the ones in the second and fourth quadrants change in the opposite direction. The response reflected by the direction and magnitude of change in septic patients agrees well with response to LPS within this common subset. However, for the SIRS group, the directions of change are not in agreement with those in endotoxemia for more than half of the metabolites. This suggests that, at the whole body metabolome level, SIRS elicits a unique response with distinctive features. One such feature is the marked decrease in sulfated androgenic hormones (epiandrosterone sulfate, androsterone sulfate, dehydroisoandrosterone sulfate (DHEA-S), 5alphapregnan-3beta,20alpha-diol disulfate) (Additional file 3: Table S2). Lower plasma concentration of one of these metabolites, DHEA-S, has previously been associated with other systemic inflammatory diseases, such as systemic lupus erythematosus and inflammatory bowel disease [36]. This supports the idea that the inflammatory response without apparent infectious stimuli might elicit distinctive features not shared with sepsis or endotoxemia. It has been previously suggested that acute inflammatory stresses from different etiologies result in highly similar responses in humans at the genomic level [37]. The observed distinct metabolomic responses to systemic inflammation with or without confirmed infection, however, suggest that the metabolome is much better at differentiating and understanding the various pathophysiologies of the different systemic inflammatory responses. Identified unique features of the inflammatory response in different contexts may aid in improving the diagnosis or the development of more targeted therapies.

Next we compared the trends of changes in metabolites within subgroups of clinical patients who ultimately survived (SS) or did not survive (SNS), and how they related with those in endotoxemia. In total, there were 78 differential metabolites between SS and SNS groups at either $t_{0}$ or $t_{24}$. Among these, 23 were also differential for the endotoxemia group at $t_{6}$. The direction and magnitude of changes in these 23 metabolites are shown in Table 3. When comparing the number of differential metabolites at either $t_{0}$ and $t_{24}$ of the SS and SNS groups, it is clear that the difference in metabolites becomes substantially more pronounced with time. Alignment of trends in the SS and SNS groups at $t_{24}$ with those in endotoxemia at $t_{6}$ revealed that the peak response to LPS is in line with the sepsis survivor metabolic response, especially at the first day into their treatment. In our previous metabolomics study [25], we identified the six hour time point as the peak of metabolic response in the endotoxemia model, 
Table 2 Metabolites which are significantly different than the heathy baseline $\left(t_{0, L P S}\right)$ in the experimental condition and either of the two time points in the clinical conditions

\begin{tabular}{|c|c|c|c|c|}
\hline \multirow{2}{*}{$\begin{array}{l}\text { A } \\
\text { Metabolite name }\end{array}$} & \multirow[b]{2}{*}{ Super pathway } & \multirow{2}{*}{$\begin{array}{l}\text { LPS } \\
\text { t6 }\end{array}$} & \multicolumn{2}{|c|}{ Sepsis } \\
\hline & & & to & t24 \\
\hline 2-hydroxybutyrate (AHB) & Amino acid & $\Delta$ & $=$ & $\boldsymbol{\Delta}$ \\
\hline mannose & Carbohydrate & $\Delta$ & $=$ & $\boldsymbol{\Delta}$ \\
\hline xylose & Carbohydrate & $\Delta$ & $=$ & $\nabla$ \\
\hline hexanoylcarnitine (C6) & Lipid & $\Delta$ & $\boldsymbol{\Delta}$ & $\boldsymbol{\Delta}$ \\
\hline bilirubin & Cofactors and vitamins & $\boldsymbol{\Delta} \boldsymbol{\Delta}$ & $\boldsymbol{\Delta}$ & $\Delta \boldsymbol{\Delta}$ \\
\hline docosapentaenoate (DPA; $22: 5 n 3$ ) & Lipid & $\boldsymbol{\Delta} \boldsymbol{\Delta}$ & $=$ & $\boldsymbol{\Delta}$ \\
\hline palmitoleate $(16: 1 \mathrm{n} 7)$ & Lipid & $\Delta \mathbf{\Delta}$ & $\boldsymbol{\Delta}$ & $\boldsymbol{\Delta}$ \\
\hline pregnen-diol disulfate & Lipid & $\Delta \boldsymbol{\Delta}$ & $\boldsymbol{\Delta}$ & $\boldsymbol{\Delta}$ \\
\hline citrulline & Amino acid & $\nabla$ & $\nabla$ & $\nabla$ \\
\hline histidine & Amino acid & $\nabla$ & $=$ & $\nabla$ \\
\hline serine & Amino acid & $\nabla$ & $=$ & $\nabla$ \\
\hline threonine & Amino acid & $\nabla$ & $\nabla$ & $=$ \\
\hline 2-palmitoyl-GPC (16:0) & Lipid & $\nabla$ & $\nabla$ & $\nabla$ \\
\hline uridine & Nucleotide & $\nabla$ & $=$ & $\nabla$ \\
\hline gamma-glutamyltyrosine & Peptide & $\nabla$ & $=$ & $\nabla$ \\
\hline catechol sulfate & Xenobiotics & $\nabla \nabla$ & $\nabla$ & $=$ \\
\hline B & & LPS & SIRS & \\
\hline Metabolite name & Super pathway & t6 & to & t24 \\
\hline alpha-ketobutyrate & Amino acid & $\Delta$ & $=$ & $\nabla$ \\
\hline $\mathrm{N}$-acetylglycine & Amino acid & $\boldsymbol{\Delta}$ & $=$ & $\nabla$ \\
\hline xylose & Carbohydrate & $\Delta$ & $=$ & $\nabla$ \\
\hline citrate & Energy & $\Delta$ & $\boldsymbol{\Delta}$ & $\boldsymbol{\Delta}$ \\
\hline arachidonate $(20: 4 n 6)$ & Lipid & $\Delta$ & $\boldsymbol{\Delta}$ & $\boldsymbol{\Delta}$ \\
\hline docosahexaenoate (DHA; 22:6n3) & Lipid & $\boldsymbol{\Delta}$ & $\boldsymbol{\Delta}$ & $=$ \\
\hline eicosapentaenoate (EPA; 20:5n3) & Lipid & $\Delta$ & $=$ & $\boldsymbol{\Delta}$ \\
\hline octadecanedioate (C18) & Lipid & $\boldsymbol{\Delta}$ & $=$ & $\nabla$ \\
\hline bilirubin & Cofactors and vitamins & $\boldsymbol{\Delta} \boldsymbol{\Delta}$ & $\boldsymbol{\Delta}$ & $=$ \\
\hline 3-hydroxybutyrate (BHBA) & Lipid & $\boldsymbol{\Delta} \boldsymbol{\Delta}$ & $=$ & $\nabla \nabla$ \\
\hline docosapentaenoate (DPA; 22:5n3) & Lipid & $\boldsymbol{\Delta} \boldsymbol{\Delta}$ & $\boldsymbol{\Delta}$ & $=$ \\
\hline hexadecanedioate (C16) & Lipid & $\boldsymbol{\Delta} \boldsymbol{\Delta}$ & $\nabla \nabla$ & $\nabla$ \\
\hline palmitoleate $(16: 1 \mathrm{n} 7)$ & Lipid & $\Delta \boldsymbol{\Delta}$ & $\boldsymbol{\Delta}$ & $\boldsymbol{\Delta}$ \\
\hline 5-oxoproline & Amino acid & $\nabla$ & $\nabla$ & $=$ \\
\hline proline & Amino acid & $\nabla$ & $=$ & $\boldsymbol{\Delta}$ \\
\hline serine & Amino acid & $\nabla$ & $\boldsymbol{\Delta}$ & $=$ \\
\hline 1-linoleoyl-GPC (18:2) & Lipid & $\nabla$ & $\boldsymbol{\Delta}$ & $\Delta$ \\
\hline 1-oleoyl-GPC (18:1) & Lipid & $\nabla$ & $=$ & $\boldsymbol{\Delta}$ \\
\hline
\end{tabular}

$\mathbf{A}$ lists the metabolites common for endotoxemia and sepsis; $\mathbf{B}$ lists those common for endotoxemia and SIRS. (=: no significant difference from $t_{0, L P S}$. $\mathbf{A} / \nabla$ : less than two fold difference from $\mathrm{t}_{0, \mathrm{LPS}} ; \boldsymbol{\Delta} \boldsymbol{\Delta} / \nabla \nabla$ : more than two fold difference from $\mathrm{t}_{0, \mathrm{LPS}}$; metabolite name in bold: common to both lists $\mathrm{A}$ and $\left.\mathrm{B}\right)$. LPS, lipopolysaccharide; SIRS, severe inflammatory response syndrome.

stating that this time point represents the point of transition from the development and recovery phases of the response. The fact that the metabolic changes in the recovering patients shift towards this response pattern strengthens the notion that the metabolic, as well as transcriptional responses, characteristic to the endotoxemia model represent necessary and 'healthy' responses to an infectious stimuli. This is further evidence of likely 
Table 3 The subset of metabolites having significantly different concentrations between SS and SNS groups at either clinical time points

\begin{tabular}{|c|c|c|c|c|c|c|}
\hline \multirow[b]{2}{*}{ Metabolite name } & \multirow[b]{2}{*}{ Super pathway } & \multicolumn{2}{|c|}{ Sepsis survivors (number $=90$ ) } & \multicolumn{2}{|c|}{ Sepsis non-survivors (number $=\mathbf{3 1}$ ) } & \multirow{2}{*}{$\begin{array}{l}\text { LPS (number }=15 \\
\text { t6 }\end{array}$} \\
\hline & & to & t24 & to & t24 & \\
\hline 2-hydroxybutyrate (AHB) & Amino acid & $\boldsymbol{\Delta}$ & - & $\Delta$ & - & $\boldsymbol{\Delta}$ \\
\hline $\mathrm{N}$-acetylglycine & Amino acid & $\nabla$ & $\nabla$ & $\boldsymbol{\Delta}$ & $\nabla$ & $\boldsymbol{\Delta}$ \\
\hline xylose & Carbohydrate & - & $\nabla$ & - & $\boldsymbol{\Delta}$ & $\boldsymbol{\Delta}$ \\
\hline malate & Energy & - & $\boldsymbol{\Delta}$ & - & $\boldsymbol{\Delta}$ & $\boldsymbol{\Delta}$ \\
\hline 10-nonadecenoate (19:1n9) & Lipid & - & $\boldsymbol{\Delta}$ & - & $\boldsymbol{\Delta}$ & $\boldsymbol{\Delta}$ \\
\hline 2-hydroxypalmitate & Lipid & $\boldsymbol{\Delta}$ & - & $\Delta$ & - & $\boldsymbol{\Delta}$ \\
\hline hexanoylcarnitine (C6) & Lipid & $\boldsymbol{\Delta}$ & $\boldsymbol{\Delta}$ & $\Delta \boldsymbol{\Delta}$ & $\boldsymbol{\Delta} \boldsymbol{\Delta}$ & $\boldsymbol{\Delta}$ \\
\hline pregn steroid monosulfate & Lipid & $\boldsymbol{\Delta}$ & - & $\nabla$ & - & $\boldsymbol{\Delta}$ \\
\hline 3-hydroxybutyrate (BHBA) & Lipid & - & $\boldsymbol{\Delta}$ & - & $\Delta \boldsymbol{\Delta}$ & $\Delta \boldsymbol{\Delta}$ \\
\hline 2-methylbutyroylcarnitine (C5) & Amino acid & $\nabla$ & $\nabla$ & $\boldsymbol{\Delta}$ & $\Delta$ & $\nabla$ \\
\hline 3-indoxyl sulfate & Amino acid & - & $\nabla$ & - & $\boldsymbol{\Delta}$ & $\nabla$ \\
\hline 5-oxoproline & Amino acid & - & $\nabla$ & - & $\boldsymbol{\Delta}$ & $\nabla$ \\
\hline histidine & Amino acid & - & $\nabla$ & - & $\nabla$ & $\nabla$ \\
\hline isobutyrylcarnitine (C4) & Am ino acid & - & $\nabla$ & - & $\Delta$ & $\nabla$ \\
\hline $\mathrm{N}$-acetylornithine & Amino acid & - & $\nabla$ & - & $\nabla \nabla$ & $\nabla$ \\
\hline tryptophan & Amino acid & - & $\nabla$ & - & $\nabla$ & $\nabla$ \\
\hline threitol & Carbohydrate & - & $\nabla$ & - & $\Delta$ & $\nabla$ \\
\hline phosphate & Energy & $\nabla$ & $\nabla$ & $\boldsymbol{\Delta}$ & $\boldsymbol{\Delta}$ & $\nabla$ \\
\hline 1-linoleoyl-GPC (18:2) & Lipid & - & $\nabla$ & - & $\nabla$ & $\nabla$ \\
\hline 1-oleoyl-GPC (18:1) & Lipid & - & $\nabla$ & - & $\nabla$ & $\nabla$ \\
\hline 2-palmitoyl-GPC (16:0) & Lipid & $\nabla$ & $\nabla$ & $\nabla \nabla$ & $\nabla \nabla$ & $\nabla$ \\
\hline propionylcarnitine (C3) & Lipid & $\boldsymbol{\Delta}$ & $\nabla$ & $\Delta$ & $\boldsymbol{\Delta}$ & $\nabla$ \\
\hline allantoin & Nucleotide & $\nabla$ & $\nabla$ & $\Delta$ & $\boldsymbol{\Delta}$ & $\nabla$ \\
\hline
\end{tabular}

Changes from the healthy baseline, $\mathrm{t}_{0, \mathrm{LPS}}: \boldsymbol{\Delta} / \nabla$ : less than two fold change; $\boldsymbol{\Delta} \boldsymbol{\Delta} / \nabla \nabla$ : more than two fold change, $-:$ there was not a significant difference between SS or SNS groups). LPS, lipopolysaccharide; SNS, sepsis non-survivors; SS, sepsis survivors.

allostatic response for survivors, that is, placing the host at the appropriate level of distress required for graceful resolution parallel to the one developed in the LPS model, versus the systemic maladaptation observed in nonsurvivors [28]. Based on this rationale, the endotoxemia model could be classified as a model of 'normal, healthy responses.' It is interesting to note that Matzinger [38] more than a decade ago proposed that the Toll-like receptors, including TLR4, evolved to serve as host defense mechanisms against major injury and trauma. Matzinger also proposed that the bacteria evolved to use this receptor system to its own advantage. This idea begins to explain why, when sufficiently controlled, LPS-induced responses might be protective and necessary rather than harmful.

The major goal of the CAPSOD study was to identify metabolite changes at sepsis presentation that predicted survival or death. Upon stratification of sepsis patients based on 28-day survival, the direction of change of 21 of 23 metabolites was the same in endotoxemia and sepsis survival (Table 3). The most important metabolite group that differentiated surviving and non-surviving CAPSOD patients was acyl-carnitines [28]. In our analysis, we observed a similar trend with all significantly changed acyl-carnitines exclusively higher than the $t_{0, L P S}$ baseline at both time points in sepsis non-survivors (Additional file 4: Table S3), whereas for the surviving patients, around half of the acyl-carnitines were below the baseline. For the endotoxemia group, the direction of change in acyl-carnitine concentrations at $t_{6, L P S}$ was the same as that of sepsis survivors (4 of total 12 acyl-carnitines were significant at $\left.t_{6, L P S}\right)$.

Finally, a number of confounding factors need to be acknowledged. Firstly, the timing of the data collection, and, therefore, the phase of the response that is being studied, can vary greatly depending on the lag time from the initiating event to the presentation to an emergency department. Secondly, the nutritional input, being non-controlled either before or after the hospital admission, could have affected the plasma metabolite concentrations as 
an independent factor. Thirdly, some of the CAPSOD patients either had prior comorbidities that were likely to affect the metabolome, such as diabetes mellitus, or were also developing conditions which further exacerbated the response, including compromised renal function, a likely major contributor to the observed metabolome.

\section{Conclusions}

Therapeutic strategies that are successfully translated into the clinic are very few and mostly non-specific in the field of critical care. This is due, in part, to the complex and dynamic physiological processes involved. Heterogeneity of the patient populations and consequent challenges in performing insightful clinical studies also have contributed to the lack of progress in this realm of medicine [3,39]. Emerging -omics tools that are capable of examining physiologic responses at the systems level are promising, especially for complex conditions, such as sepsis and SIRS [40]. The major caveat related to these tools is that since the biological processes are analyzed at a higher level, inter-species differences become as relevant to the response as the sought-after question itself. Therefore, utility of the animal models has been questioned recently in the scientific community [37,41].

The human endotoxemia model has been serving as a useful experimental platform for gaining insight into the mechanisms governing systemic inflammation. It is a recognized fact that this model does not fully replicate the magnitude of physiologic stress created by trauma or infection [13,14]; however, it gives researchers the opportunity to study the mechanisms underlying the response to systemic inflammation and relevant therapy options without the inter-species differences obscuring the interpretation of the results.

Progression of response to systemic inflammation induced by endotoxemia in immune cells has been described at the genomic level $[16,30]$. Moreover, comparison of the response to experimental stimuli and traumatic/infectious insults revealed significant overlap of common features both at the gene [15] and protein expression levels [34]. In the light of these observations, the current study aimed at an objective evaluation of the concordance between experimental and clinical cases of systemic inflammation and benchmarked endotoxemia against sepsis of various origins at the level of metabolic response. The plasma metabolome can be thought of as the metabolic fingerprint representative of the state of the body at any given time and provide information on the dominant regulatory mechanisms at various levels of cellular processes including transcription, translation and signal transduction. For effective provision of critical care, understanding the alterations in the plasma metabolome is crucial, because metabolite levels impact the regulation of anti-inflammatory defenses, in turn, through steering critical cellular processes and immune mechanisms. Therefore, we think that the assessment of the relevance of endotoxemia as an experimental model representing critical illness is important.

We believe that the observed concordance between the responses of LPS-treated subjects and sepsis patients at the metabolome level, despite observed variability in clinical data, strengthens the relevance of endotoxemia to clinical research as an elementary tool and gives valuable insights into the metabolic changes necessary for proper response to inflammatory stress at the systemic level.

\section{Key messages}

- We compared the metabolic response at the peak of LPS-induced acute inflammation with those from sepsis patients

- For the metabolites shown to change significantly from the baseline, the direction and magnitude of the changes were in agreement with what was observed in sepsis patients.

- The metabolic response in SIRS patients was shown be distinct from those in endotoxemia or sepsis.

- Metabolic changes in the surviving sepsis patients shifted towards those observed in endotoxemia as their recovery proceeded.

- These observations strengthened the relevance of endotoxemia to clinical research as a valuable experimental tool which can enable further research on the development and assessment of rational clinical therapies to prevent sepsis mortality.

\section{Additional files}

Additional file 1: Table S1. Number of outliers removed from the data before any statistical analysis.

Additional file 2: Figure S1. Comparison of the variances of significant metabolites in the clinical groups with respect to those in the baseline ( $\left.\mathrm{t}_{0, L P S}\right)$.

Additional file 3: Table S2. Full list of the metabolites, their significance in each condition, direction and magnitude of the changes relative to the baseline $\left(\mathrm{t}_{0, \mathrm{LPS}}\right)$.

Additional file 4: Table S3. Results of t-test for acyl-GPCS and acyl-carnitines, between SS and SNS groups at each time point, and between $t_{0, L P S}$ and $t_{6, L P S}$ in endotoxemia group, together with their direction of change from the common baseline $t_{0, L P S}$. (Changes from the healthy baseline, $\mathrm{t}_{0, \mathrm{LPS}}: \boldsymbol{\Delta} / \nabla$ : less than 2 fold change; $\boldsymbol{\Delta} \boldsymbol{\Delta} / \nabla \nabla$ : more than 2 fold change).

\section{Abbreviations}

APACHE II: Acute Physiology and Chronic Health Evaluation II; CAPSOD: Community Acquired Pneumonia and Sepsis Outcome and Diagnostics study; LPS: lipopolysaccharide; MAD: median absolute deviation; MS: mass spectrometry; SIRS: systemic inflammatory response syndrome; SNS: sepsis non-survivors; SOFA: Sequential Organ Failure Assessment; SS: sepsis survivors; TLR4: Toll-like receptor 4.

\section{Competing interests}

The authors declare that they have no competing interests. 


\section{Authors' contributions}

KK performed the analysis and prepared the manuscript. BH, SEC, SMC, SAC assisted with the design of experimental endotoxemia and edited the manuscript. RJL and SFK provided the patient data and edited the manuscript IPA designed and oversaw the analysis and edited the final manuscript. All authors read and approved the final manuscript.

\section{Acknowledgements}

The authors greatly acknowledge the financial support from the National Institutes of Health (NIH) (Grants: GM082974, GM34695, and U01Al066569), Pfizer Inc., and Roche Diagnostics Inc.

\section{Author details}

'Department of Chemical and Biochemical Engineering, Rutgers University, Piscataway, NJ 08854, USA. ²Department of Surgery, Rutgers - Robert Wood Johnson Medical School, New Brunswick, NJ 08901, USA. ${ }^{3}$ Department of Respiratory Immunology, Lovelace Respiratory Research Institute, Albuquerque, NM 87108, USA. ${ }^{4}$ Center for Pediatric Genomic Medicine, Children's Mercy, Kansas City, MO 64108, USA. ${ }^{5}$ Departments of Pediatrics and Obstetrics/Gynecology, University of Missouri, Kansas City, MO 64108 , USA. ${ }^{6}$ Department of Biomedical Engineering, Rutgers University, 599 Taylor Road, Piscataway, NJ 08854, USA.

Received: 23 July 2014 Accepted: 3 February 2015

Published online: 03 March 2015

\section{References}

1. Levy MM, Fink MP, Marshall JC, Abraham E, Angus D, Cook D, et al. 2001 SCCM/ESICM/ACCP/ATS/SIS International Sepsis Definitions Conference. Crit Care Med. 2003;31:1250-6.

2. Hall MJ, Williams SN, DeFrances CJ, Golosinskiy A. Inpatient care for septicemia or sepsis: a challenge for patients and hospitals. NCHS Data Brief. 2011;62:1-8.

3. Angus DC, van der Poll T. Severe sepsis and septic shock. N Engl J Med. 2013;369:840-51.

4. Lagu T, Rothberg MB, Shieh MS, Pekow PS, Steingrub JS, Lindenauer PK Hospitalizations, costs, and outcomes of severe sepsis in the United States 2003 to 2007. Crit Care Med. 2012:40:754-61.

5. Reinhart K, Bauer M, Riedemann NC, Hartog CS. New approaches to sepsis: molecular diagnostics and biomarkers. Clin Microbiol Rev. 2012;25:609-34.

6. Rittirsch D, Hoesel LM, Ward PA. The disconnect between animal models of sepsis and human sepsis. J Leukoc Biol. 2007;81:137-43.

7. Deitch EA. Animal models of sepsis and shock: a review and lessons learned. Shock. 1998;9:1-11.

8. Buras JA, Holzmann B, Sitkovsky M. Animal models of sepsis: setting the stage. Nat Rev Drug Discov. 2005;4:854-65.

9. Munford RS. Detoxifying endotoxin: time, place and person. J Endotoxin Res. 2005;11:69-84.

10. Wolff SM. Biological effects of bacterial endotoxins in man. J Infect Dis. 1973;128:259-64.

11. Andreasen AS, Krabbe KS, Krogh-Madsen R, Taudorf S, Pedersen BK, Moller K. Human endotoxemia as a model of systemic inflammation. Curr Med Chem. 2008;15:1697-705

12. Lin E, Lowry S. The human response to endotoxin. Sepsis. 1998:2:255-62.

13. Lowry SF. Human endotoxemia: a model for mechanistic insight and therapeutic targeting. Shock. 2005:24:94-100.

14. Calvano SE, Coyle SM. Experimental human endotoxemia: a model of the systemic inflammatory response syndrome? Surg Infect (Larchmt). 2012:13:293-9.

15. Haimovich B, Reddell MT, Calvano JE, Calvano SE, Macor MA, Coyle SM, et al. A novel model of common Toll-like receptor 4- and injury-induced transcriptional themes in human leukocytes. Crit Care. 2010;14:R177.

16. Calvano SE, Xiao W, Richards DR, Felciano RM, Baker HV, Cho RJ, et al. A network-based analysis of systemic inflammation in humans. Nature. 2005;437:1032-7.

17. Nguyen TT, Foteinou PT, Calvano SE, Lowry SF, Androulakis IP. Computational identification of transcriptional regulators in human endotoxemia. PLoS One. 2011:6:e18889.

18. Foteinou P, Calvano S, Lowry S, Androulakis I. Modeling endotoxin-induced systemic inflammation using an indirect response approach. Math Biosci. 2009;217:27-42.

19. Foteinou PT, Calvano SE, Lowry SF, Androulakis IP. A physiological model for autonomic heart rate regulation in human endotoxemia. Shock. 2011;35:229.
20. Scheff JD, Mavroudis PD, Calvano SE, Lowry SF, Androulakis IP. Modeling autonomic regulation of cardiac function and heart rate variability in human endotoxemia. Physiol Genomics. 2011;43:951-64.

21. Foteinou PT, Calvano SE, Lowry SF, Androulakis IP. Multiscale model for the assessment of autonomic dysfunction in human endotoxemia. Physiol Genomics. 2010;42:5-19.

22. Scheff JD, Mavroudis PD, Foteinou PT, Calvano SE, Androulakis IP. Modeling physiologic variability in human endotoxemia. Crit Rev Biomed Eng. 2012;40:313-22.

23. Scheff JD, Calvano SE, Lowry SF, Androulakis IP. Modeling the influence of circadian rhythms on the acute inflammatory response. J Theor Biol. 2010;264:1068-76.

24. Scheff JD, Mavroudis PD, Calvano SE, Androulakis IP. Translational applications of evaluating physiologic variability in human endotoxemia. J Clin Monit Comput. 2013:27:405-15.

25. Kamisoglu K, Sleight KE, Calvano SE, Coyle SM, Corbett SA, Androulakis IP. Temporal metabolic profiling of plasma during endotoxemia in humans. Shock. 2013;40:519-26

26. Kosmides AK, Kamisoglu K, Calvano SE, Corbett SA, Androulakis IP. Metabolomic fingerprinting: challenges and opportunities. Crit Rev Biomed Eng. 2013:41:205-21.

27. Kamisoglu K, Calvano SE, Coyle S, Corbett SA, Androulakis IP. Integrated transcriptional and metabolic profiling in human endotoxemia. Shock. 2014:42:499-508.

28. Langley RJ, Tsalik EL, van Velkinburgh JC, Glickman SW, Rice BJ, Wang C, et al. An integrated clinico-metabolomic model improves prediction of death in sepsis. Sci Transl Med. 2013;5:195ra195.

29. Evans AM, DeHaven CD, Barrett T, Mitchell M, Milgram E. Integrated, nontargeted ultrahigh performance liquid chromatography/electrospray ionization tandem mass spectrometry platform for the identification and relative quantification of the small-molecule complement of biological systems. Anal Chem. 2009:81:6656-67.

30. Talwar S, Munson PJ, Barb J, Fiuza C, Cintron AP, Logun C, et al. Gene expression profiles of peripheral blood leukocytes after endotoxin challenge in humans. Physiol Genomics. 2006:25:203-15.

31. Leys C, Ley C, Klein O, Bernard P, Licata L. Detecting outliers: do not use standard deviation around the mean, use absolute deviation around the median. J Exp Soc Psychol. 2013;49:764-6.

32. Hampel FR. The influence curve and its role in robust estimation. J Am Stat Assoc. 1974;69:383-93.

33. Benjamini $Y$, Hochberg $Y$. Controlling the false discovery rate: a practical and powerful approach to multiple testing. J R Stat Soc Ser B Methodol. 1995;57:289-300

34. Visser T, Pillay J, Pickkers P, Leenen LP, Koenderman L. Homology in systemic neutrophil response induced by human experimental endotoxemia and by trauma. Shock. 2012;37:145-51.

35. Glickman SW, Cairns CB, Otero RM, Woods CW, Tsalik EL, Langley RJ, et al. Disease progression in hemodynamically stable patients presenting to the emergency department with sepsis. Acad Emerg Med. 2010;17:383-90.

36. Straub RH, Vogl D, Gross V, Lang B, Scholmerich J, Andus T. Association of humoral markers of inflammation and dehydroepiandrosterone sulfate or cortisol serum levels in patients with chronic inflammatory bowel disease. Am J Gastroenterol. 1998:93:2197-202.

37. Seok J, Warren HS, Cuenca AG, Mindrinos MN, Baker HV, Xu W, et al. Genomic responses in mouse models poorly mimic human inflammatory diseases. Proc Natl Acad Sci U S A. 2013;110:3507-12.

38. Matzinger $P$. The danger model: a renewed sense of self. Science. 2002;296:301-5.

39. Cain D, del Arroyo A, Ackland G. Uncontrolled sepsis: a systematic review of translational immunology studies in intensive care medicine. ICMx. 2014;2:1-25.

40. Maslove DM, Wong HR. Gene expression profiling in sepsis: timing, tissue, and translational considerations. Trends Mol Med. 2014;20:204-13.

41. Osuchowski MF, Remick DG, Lederer JA, Lang CH, Aasen AO, Aibiki M, et al. Abandon the mouse research ship? Not just yet! Shock. 2014:41:463-75. 